University of Wollongong

Research Online

Illawarra Health and Medical Research Institute

Faculty of Science, Medicine and Health

January 2015

\title{
Fatty acid composition of the anterior cingulate cortex indicates a high susceptibility to lipid peroxidation in Parkinson's disease
}

\author{
Sarah K. Abbott \\ University of Wollongong, sarahmac@uow.edu.au \\ Andrew M. Jenner \\ University of Wollongong, ajenner@uow.edu.au \\ Adena S. Spiro \\ University of Wollongong, adena@uow.edu.au \\ Marijka Batterham \\ University of Wollongong, marijka@uow.edu.au \\ Glenda M. Halliday \\ University of New South Wales
}

See next page for additional authors

Follow this and additional works at: https://ro.uow.edu.au/ihmri

Part of the Medicine and Health Sciences Commons

\section{Recommended Citation}

Abbott, Sarah K.; Jenner, Andrew M.; Spiro, Adena S.; Batterham, Marijka; Halliday, Glenda M.; and Garner, Brett, "Fatty acid composition of the anterior cingulate cortex indicates a high susceptibility to lipid peroxidation in Parkinson's disease" (2015). Illawarra Health and Medical Research Institute. 496.

https://ro.uow.edu.au/ihmri/496

Research Online is the open access institutional repository for the University of Wollongong. For further information contact the UOW Library: research-pubs@uow.edu.au 


\title{
Fatty acid composition of the anterior cingulate cortex indicates a high susceptibility to lipid peroxidation in Parkinson's disease
}

\author{
Abstract \\ Background: Oxidative stress contributes to Parkinson's disease (PD) etiology. Although previous studies \\ have focused on sources of free radical formation in brain regions affected by PD, less is known regarding \\ changes in lipid composition and the implications for susceptibility to peroxidation. Objective: To assess \\ fatty acid profiles from control and PD tissues that are susceptible to PD pathology but devoid of severe \\ destruction. Methods: We used gas chromatography methods to assess fatty acid profiles from control ( $n$ \\ $=10)$ and $P D(n=9)$ postmortem tissues. We focused on the anterior cingulate cortex (ACC), a region that \\ accumulates alpha-synuclein, but does not undergo severe destruction, and compared this to the occipital \\ cortex, a region that is pathologically spared. Results: Our data indicate a significant $33 \%$ increase in the \\ proportion of polyunsaturated fatty acids (mol\%) present in the PD ACC as compared to control ACC. \\ Increases in highly unsaturated 22:5n-6 and 22:6n-3 fatty acids were particularly pronounced (109\% and \\ $73 \%$, respectively). Calculation of a peroxidation index (accounting for total fatty acyl double bounds) \\ indicated a $44 \%$ increase in susceptibility of the PD ACC to lipid peroxidation compared to control ACC. \\ Such differences were not detected in the occipital cortex from the same donors. Assessment of \\ F2-isprostane levels confirmed that PD tissue lipids were more oxidized than controls. Conclusions: The \\ global composition of fatty acids in the PD ACC is altered in a way that increases susceptibility to \\ peroxidation in a region-specific manner. This has important implications for PD, supporting the oxidative \\ stress hypothesis of PD pathogenesis.

\section{Disciplines} \\ Medicine and Health Sciences

\section{Publication Details} \\ Abbott, S. K., Jenner, A. M., Spiro, A. S., Batterham, M., Halliday, G. M. \& Garner, B. (2015). Fatty acid \\ composition of the anterior cingulate cortex indicates a high susceptibility to lipid peroxidation in \\ Parkinson's disease. Journal of Parkinson's Disease, 5 (1), 175-185.

\section{Authors} \\ Sarah K. Abbott, Andrew M. Jenner, Adena S. Spiro, Marijka Batterham, Glenda M. Halliday, and Brett \\ Garner
}


Fatty acid composition of the anterior cingulate cortex indicates a high susceptibility to lipid peroxidation in Parkinson's disease

Sarah K. Abbott ${ }^{\text {a,b, }}$, Andrew M. Jenner ${ }^{a, b}$, Adena S. Spiro ${ }^{\text {a,b }}$, Marijka Batterham ${ }^{\text {c }}$,

Glenda M. Halliday ${ }^{\mathrm{d}}$, Brett Garner ${ }^{\mathrm{a}, \mathrm{b}, *}$

a Illawarra Health and Medical Research Institute, University of Wollongong, NSW 2522, Australia

${ }^{\mathrm{b}}$ School of Biological Sciences, University of Wollongong, NSW 2522, Australia

${ }^{c}$ National Institute of Applied Statistics Research Australia, University of Wollongong, Wollongong NSW 2522, Australia

${ }^{\mathrm{d}}$ Neuroscience Research Australia and the University of New South Wales, Sydney, NSW 2031, Australia

Running title: Fatty acid profiles in Parkinson's disease

\section{Correspondence to}

Dr Sarah Abbott, Illawarra Health and Medical Research Institute, University of Wollongong, NSW 2522, Australia, Email: sarahmac@uow.edu.au; Tel: +61 24298 1997; Fax: +61 2 42218130

or

Prof Brett Garner, Illawarra Health and Medical Research Institute, University of Wollongong, NSW 2522, Australia; Email: brettg@uow.edu.au; Tel: +61 24298 1576; Fax: $+61242218130$. 


\section{ABSTRACT}

BACKGROUND: Oxidative stress contributes to Parkinson's disease (PD) etiology.

Although previous studies have focused on sources of free radical formation in brain regions

affected by PD, less is known regarding changes in lipid composition and the implications for susceptibility to peroxidation.

OBJECTIVE: To assess fatty acid profiles from control and PD tissues that are susceptible to PD pathology but devoid of severe destruction.

METHODS: We used gas chromatography methods to assess fatty acid profiles from control $(n=10)$ and PD $(n=9)$ postmortem tissues. We focused on the anterior cingulate cortex (ACC), a region that accumulates alpha-synuclein, but does not undergo severe destruction, and compared this to the occipital cortex, a region that is pathologically spared.

RESULTS: Our data indicate a significant 33\% increase in the proportion of polyunsaturated fatty acids (mol\%) present in the PD ACC as compared to control ACC. Increases in highly unsaturated 22:5n-6 and 22:6n-3 fatty acids were particularly pronounced (109\% and $73 \%$, respectively). Calculation of a peroxidation index (accounting for total fatty acyl double bounds) indicated a $44 \%$ increase in susceptibility of the PD ACC to lipid peroxidation compared to control ACC. Such differences were not detected in the occipital cortex from the same donors. Assessment of $\mathrm{F}_{2}$-isprostane levels confirmed that $\mathrm{PD}$ tissue lipids were more oxidized than controls.

CONCLUSIONS: The global composition of fatty acids in the PD ACC is altered in a way that increases susceptibility to peroxidation in a region-specific manner. This has important implications for PD, supporting the oxidative stress hypothesis of PD pathogenesis. 
Key words: Parkinson's disease, fatty acids, lipid peroxidation, F2-isoprostanes, oxidative stress, postmortem tissue analysis

\section{Abbreviations}

ACC, anterior cingulate cortex; $\alpha$-syn, alpha-synuclein; BHT, butylated hydroxytoluene;

Con, control; $F_{2}$-ISPs, $F_{2}$-isoprostanes; MTBE, methyl-tert-butyl ether; OCC, occipital

cortex; PD, Parkinson's disease; PI, peroxidation index; PUFA, polyunsaturated fatty acid;

SN, substantia nigra pars compacta 


\section{INTRODUCTION}

Parkinson's disease (PD) affects approximately 1-2\% of the population over 65 years increasing to $3-5 \%$ in people over 85 years old $[1,2]$. The pathological hallmarks of PD include the loss of dopaminergic neurons in the substantia nigra pars compacta (SN) and the formation of Lewy bodies and Lewy neurites that are composed of aggregated alphasynuclein $(\alpha$-syn) and other components including lipids [3,4,5]. Lewy pathologies occur progressively in selected neurons located in the brainstem, limbic cortices and finally the neocortex $[3,6,7,8]$. Degeneration of the SN and involvement of the amygdala occur preclinically with the anterior cingulate cortex (ACC) affected early after the onset of clinical symptoms, whereas the occipital cortex (OCC) is pathologically spared $[3,6,7,8]$.

There is very strong evidence indicating that oxidative stress is associated with PD pathology $[9,10,11,12]$. Several studies have suggested that lipid peroxidation may represent a significant etiological factor and accelerator of disease progression $[11,12,13,14,15]$. Many lines of investigation have been pursued related to oxidative stress in PD, and these have most commonly focused on identifying the origin of free radical species, including the roles of tyrosine, DOPA and tyrosine hydroxylase; iron, copper and metal-rich substances, such as neuromelanin; dysfunctions in mitochondrial electron transport, and protein / amino acid radicals, for example related to $\alpha$-syn Met side chains, and amino acid-metal complexes $[16,17,18]$

A selective decrease in antioxidant "defense" systems, including altered GSH metabolism, decreased ferritin, and changes in levels of vitamins $\mathrm{C}$ and $\mathrm{E}$, polyphenols and coenzyme Q10, have also been postulated to be associated with, and to predispose towards, 
neurodegeneration in PD [19,20,21]. It is generally thought that an imbalance between free radical production and antioxidant defense increases oxidative stress and thereby contributes to PD pathology $[11,12]$. This is supported by studies showing region-specific increases in oxidative stress in the PD brain. For example, previous studies have shown that the $\mathrm{SN}$ is subjected to oxidative stress $[16,17,18,22,23,24]$. Other regions that may also be affected by Lewy pathology albeit less severely (e.g. frontal cortex, putamen and caudate) also appear to be associated with oxidative stress, although this varies depending on the precise markers of oxidative stress / damage assessed $[13,14,15,25]$. Another finding replicated in several studies is that aggregation of $\alpha$-syn is generally associated with lipid peroxidation $[14,26,27,28,29,30]$; whether this is due to a failed antioxidant action of $\alpha$-syn or a detrimental action of $\alpha$-syn / $\alpha$-syn-metal complexes remains to be determined.

An additional important factor that has not received as much attention relates to the global fatty acid composition of brain tissues and the calculated propensity of such fatty acids to become oxidized. This is important, as it is well known that a higher level of lipid acyl chain unsaturation is a very strong indicator of tissue/membrane susceptibility to peroxidation $[31,32,33]$. Indeed, the "oxidizabilities" of polyunsaturated fatty acids (PUFAs) are linearly dependent on the number of double bonds in the fatty acyl chains [32]. It is therefore possible to calculate a peroxidation index (PI) based on the number of fatty acid bisallylic double bonds in model systems and in animal tissues [31,33]. Furthermore, there is increasing evidence that a higher membrane PUFA content (i.e. higher PI) contributes to membrane lipid oxidation and an inverse relationship with longevity across a wide range of animal species [34]. Even though there may be a rationale for certain health-promoting benefits of specific PUFAs in the PD context [35], it is critical to understand how tissue PUFA balance might be altered in PD and how such changes may associate with lipid peroxidation in the 
brain. In the present study we have addressed this issue by undertaking a detailed study of global fatty acid profiles in the ACC and OCC regions of healthy control and confirmed PD cases. We have previously shown in the same cases a significant shift in ceramide function in lipid membranes and signaling pathways in the PD ACC [36]. In the present study we calculate PI values and $\mathrm{F}_{2}$-isoprostane $\left(\mathrm{F}_{2}\right.$-ISP) levels as markers of lipid peroxidation and reveal that the PD ACC fatty acid profile has an increased susceptibility to peroxidation as compared to control tissues.

\section{MATERIALS AND METHODS}

\section{Materials}

Methyl-tert-butyl ether (MTBE), chloroform and methanol were HPLC grade and purchased from Thermo Scientific, Scoresby, VIC, Australia. Analytical grade butylated hydroxytoluene (BHT) and sodium hydroxide (98\% minimum) were from Sigma Aldrich, Sydney, NSW, Australia and analytical grade ammonium acetate was from Crown Scientific, Moorebank, NSW, Australia. Screw thread vials (4 ml) and wide mouth vials (1.8 ml) with PTFE/silicone septa caps were from Grace Davison, Rowville, VIC, Australia. The fatty acid standard contained heneicosanoic acid (C21:0; $3.1 \mathrm{mM}$ in methanol; purchased from Sigma Aldrich, Sydney, NSW, Australia) The $\mathrm{F}_{2}$-ISP internal standards were obtained from Cayman chemicals (Sapphire Bioscience, Sydney, NSW, Australia) and were dissolved in ispopropanol as previously described [37]. All lipid internal standards were stored at $-20^{\circ} \mathrm{C}$.

\section{Human brain tissue}

Frozen brain grey matter from 10 control cases and 9 sporadic PD cases were received from the Sydney Brain Bank and the NSW Tissue Resource Centre. Standardized 
clinicopathological criteria were used for diagnosis with no case or control having any evidence of significant neuropathology other than PD [38]. This cohort of control and PD tissues has been previously described in detail [36]. The control and PD groups were matched for both age $($ Con $74.7 \pm 2.9 \mathrm{y}, \mathrm{PD} 77.8 \pm 1.9 \mathrm{y}$, mean $\pm \mathrm{SE})$ and postmortem interval $(\mathrm{Con}$ $22.0 \pm 4.8 \mathrm{~h}, \mathrm{PD} 18.4 \pm 2.9 \mathrm{~h}$, mean $\pm \mathrm{SE}$ ). Tissue $\mathrm{pH}$ levels were all $>6.0$ and there were no significant differences $(\mathrm{P}>0.05)$ between the control and $\mathrm{PD}$ groups [36]. The PD cases all had increased $\alpha$-syn levels in the ACC whereas such an increase was not present in the control ACC nor in the PD OCC or control OCC [36]. The ACC Lewy body score was based on $\alpha$-syn pathology and was measured using immunohistochemical techniques as previously reported [36]. In addition, the levels of SDS-soluble $\alpha$-syn present in the PD ACC were previously quantified by western blotting as described and reported [39]. The relative optical density value for the SDS-soluble $\alpha$-syn level in all PD ACC samples was used to assess $\alpha$ syn levels. Ethics approval was from the University of New South Wales Human Research Ethics Committee and the University of Wollongong Human Research Ethics Committee. The research has been carried out in accordance with the Declaration of Helsinki (2008) of the World Medical Association. All persons gave their informed consent prior to their inclusion in the study.

Approximately $35 \mathrm{mg}$ of frozen brain tissue from the ACC and OCC was pulverized over dry ice and two aliquots of approximately 20 and $15 \mathrm{mg}$ were accurately weighed and stored at $80^{\circ} \mathrm{C}$ until required for fatty acid and $\mathrm{F}_{2}$-ISP analysis, respectively.

\section{Lipid extraction}

The lipid extraction was performed as previously described in detail [40]. In brief, approximately $15 \mathrm{mg}$ of pulverised human brain tissue was accurately weighed directly into 
each $0.5 \mathrm{ml}$ Precellys ${ }^{\circledR}$ tube (Sapphire Bioscience, Waterloo, NSW, Australia) and homogenised in $300 \mu 1$ ice-cold methanol containing fatty acid/ $\mathrm{F}_{2}$-ISP internal standards $(2 \mu \mathrm{l}$ /mg tissue and $10 \mu \mathrm{l} /$ sample, respectively) and BHT (0.01\% w/v), using a Precellys ${ }^{\circledR} 24$ bead homogeniser (Bertin Technologies; 2 x $30 \mathrm{sec}$ at $6000 \mathrm{rpm}$ ). After transferring the

homogenate to a $4 \mathrm{ml}$ glass vial, the Precellys tube was rinsed with $162 \mu 1$ methanol and 1540 $\mu 1$ MTBE was added to the glass vial (final MTBE/methanol ratio 10:3, vol:vol). Following overnight extraction, $380 \mu \mathrm{l}$ of $0.15 \mathrm{M}$ ammonium acetate was added and the organic (upper) phase was collected in a $4 \mathrm{ml}$ vial. The original homogenate was re-extracted by addition of $616 \mu 1$ of the upper phase MTBE/methanol/0.15M ammonium acetate and combining the upper phase with the first extract. This lipid extract was then dried under nitrogen at $37^{\circ} \mathrm{C}$ and resuspended in chloroform/methanol (1:2, vol:vol).

\section{Analysis of fatty acid methyl esters by GC}

Fatty acids were methylated and analysed as fatty acid methyl esters by gas chromatography (GC) as described previously [41]. Susceptibility of lipid composition to oxidation was determined using an established peroxidation index (PI) as described previously [32]. In brief, relative contributions of the fatty acid classes were used in the formula: $\mathrm{PI}=(0.025 \%$ monoenoics $)+(1 \%$ dienoics $)+(2 \%$ trienoics $)+(4 \%$ tetraenoics $)+(6 \%$ pentaenoics $)+(8 \%$ hexaenoics) [32].

\section{Analysis of $\mathrm{F}_{2}$-isoprostanes by GC-MS}

Lipid extracts (from $15 \mathrm{mg}$ tissue) were hydrolysed overnight for GC-MS analysis of $\mathrm{F}_{2}$-ISPs as described previously [37]. Samples were loaded onto pre-conditioned solid phase extraction columns (UCT CUQAX223 3 ml; United Chemical Technologies, Bristol, USA) and washed with $2 \mathrm{ml}$ of $40 \mathrm{mM}$ formic acid ( $\mathrm{pH} 4.5)$ containing $40 \%(\mathrm{v} / \mathrm{v})$ methanol and 2 
$\mathrm{ml}$ hexane/MTBE (1:1). $\mathrm{F}_{2}$-isoprostanes and fatty acids were eluted from the SPE columns with MTBE containing $20 \%$ methanol and $1 \%$ formic acid. The $\mathrm{F}_{2}$-ISP and fatty acid fraction was dried down under nitrogen and derivatised with $30 \mu 1$ of pentafluorobenzylbromide (PFBBr) $(10 \%$ in acetonitrile) and $15 \mu 1$ of N,N-diisopropylethylamine (DIPEA) $(10 \%$ in acetonitrile) at $37^{\circ} \mathrm{C}$ for $30 \mathrm{~min}$. Excess reagents were evaporated under nitrogen. The $\mathrm{F}_{2}$-ISP PFBenzyl ester was derivatised with $15 \mu \mathrm{l}$ acetonitrile plus $30 \mu \mathrm{l}$ BSTFA $+1 \%$ TMCS for 1 hour at $37^{\circ} \mathrm{C}$. The derivatised samples were dried under nitrogen, reconstituted in $30 \mu 1$ toluene and analysed using the GC-MS. Selected-reaction monitoring (SRM) was performed using the negative chemical ionisation (NCI) mode $(70 \mathrm{eV})$ with argon as the reagent gas $(1.25 \mathrm{ml} / \mathrm{min})$ and the collision gas $(0.6 \mathrm{ml} / \mathrm{min})$. The ion source was maintained at $150^{\circ} \mathrm{C}$ and the quadrupoles at $150^{\circ} \mathrm{C}$. For $\mathrm{F}_{2}$-ISP analysis, derivatised samples $(1 \mu \mathrm{l})$ were injected splitless into the $\mathrm{GC}$ injection port. Column temperature was increased from $180^{\circ} \mathrm{C}$ to $280^{\circ} \mathrm{C}$ at $40^{\circ} \mathrm{C} / \mathrm{min}$ to $305^{\circ} \mathrm{C}$ with a final hold of $4 \mathrm{~min}$. Quantification of $\mathrm{F}_{2}$-ISPs $\left(\mathrm{F}_{2} \mathrm{\alpha III}-\mathrm{ISP}+\right.$ $\mathrm{F}_{2} \alpha$ VI-ISP) was achieved by comparison of specific SRM transitions with their corresponding heavy isotope internal standards.

\section{Statistical analysis}

Differences between the Control and PD group fatty acids were assessed using a 2-tailed ttest for unpaired data where $\mathrm{P}<0.05$ was considered significant. Additional post hoc comparisons were conducted using the method of Benjamini and Hochberg [42], these analyses were conducted using the p.adjust command in the package "stats" in R version 2.15.2 (R Core Development Team)[43]. Differences in $\mathrm{F}_{2}$-ISPs were assessed in a separate sample using one-way ANOVA and post-hoc analysis by Fisher's Least Significant Difference (LSD) comparison where $\mathrm{P}<0.05$ was considered significant. 


\section{RESULTS}

\section{Fatty acid analysis in the anterior cingulate cortex}

In the current study we compared global fatty acid profiles in the ACC and OCC. The ACC was used as pathological changes associated with $\alpha$-syn are present from Braak stage 4 $[3,6,7,8]$, whereas the OCC was chosen as it is pathologically spared and serves as a regionspecific comparator $[44,45]$. Other regions of the brain that may be more severely affected by PD pathology (e.g. the SN) were avoided as accompanying loss of neuronal structures may potentially change fatty acid profiles due to gross alterations in cellular / tissue composition in PD cases as compared to control tissues.

Analysis of fatty acid methyl esters by GC revealed 15 predominant lipid species. The most abundant fatty acids in the ACC were C16:0, C18:0 and C18:1n9. When the fatty acid levels were quantified and expressed per mg brain tissue, most species were significantly lower in the PD ACC compared to the Con ACC (Table 1). Overall fatty acid levels were $26.5 \%$ lower in the PD ACC compared to the Con ACC, a finding consistent with our recent data related to sphingolipid changes in the PD brain [36]. More importantly, in terms of assessing propensity to lipid peroxidation, when the fatty acids were compared in terms of their mol\% distribution, we detected a significant change in the relative proportion of saturated and unsaturated lipids in the PD ACC compared to the Con ACC (Fig 1A). Notably, the relative amounts of the two fatty acids with the greatest degree of unsaturation (C22:5n 6 an $\mathrm{C} 22: 6 \mathrm{n} 3)$ were increased by $109 \%$ and $73 \%$, respectively (Fig 1A). As a fatty acid class, the relative concentration of PUFAs was increased by $33 \%$ in the PD ACC compared to the Con ACC, and this resulted in a significant $44 \%$ increase in the calculated PI (Fig 1B). This analysis reveals for the first 
time that the PD ACC has a greater propensity for lipid peroxidation compared to the Con ACC

\section{Fatty acid analysis in the occipital cortex}

Based on previous data showing that Lewy body pathology is associated with lipid peroxidation $[14,46]$ and our recent study showing that the PD cohort used in the current experiments have increased levels of insoluble $\alpha$-syn in the ACC [36], we also assessed the OCC as a comparator region that is largely devoid of pathology. The overall levels of fatty acids detected in the OCC were similar to the ACC (Table 2). In contrast, there was only one statistically significant change; levels of $\mathrm{C} 16: 1 \mathrm{n} 7$ were $22.6 \%$ lower in the PD OCC compared to the Con OCC (Table 2). Although fatty acid levels were not different overall, a trend $(\mathrm{P}=0.07)$ for a $20 \%$ reduction in fatty acids in the PD OCC compared to the Con OCC was noted (Table 2).

When the fatty acids were compared in terms of their mol $\%$ distribution, the overall profiles were quite similar to those observed in both the PD ACC and Con ACC samples (Figs 1A and 2A), however, in contrast to this similarity, there were no differences in the proportional distribution of fatty acids, their degree of unsaturation, or the PI values when the PD OCC was compared to the Con OCC (Fig 2B). This indicates that region-specific changes in global fatty acid profiles are associated with PD.

\section{$F_{2}$-isoprostane analysis}

In order to determine whether the change in PUFA distribution and PI observed in the PD ACC was associated with a marker of lipid oxidation, we assessed tissue $\mathrm{F}_{2}$-ISP levels by GC-MS. The data indicate significant between group interactions by one-way ANOVA ( $\mathrm{P}=$ 
0.0003). Post-hoc analysis by Fisher's LSD revealed a significant $(P=0.005) 72 \%$ increase in $\mathrm{F}_{2}$-ISP levels in the PD ACC compared to the Con ACC (Fig 3). We also detected a trend for an increase in $\mathrm{OCC} \mathrm{F}_{2}$-ISP levels when the PD group was compared to the Con group (P $=0.053)$. In addition, the PD OCC $F_{2}$-ISP levels were significantly $(\mathrm{P}=0.005)$ lower (by $46 \%$ ) than the amount detected in the PD ACC. Overall, this indicates a significant increase in lipid peroxidation in PD that is more pronounced in the ACC, a region with increased $\alpha$ syn aggregation.

\section{DISCUSSION}

The vast majority of brain tissue fatty acids $(>95 \%)$ we have analysed herein are derived from membrane phospholipids. The extent of fatty acyl chain saturation is well known to affect membrane fluidity, and specific fatty acids have potent biological activities and/or give rise to biologically active molecules $[35,47]$. For example, arachidonic acid (C20:4n-6) is the precursor for prostaglandins, leukotrienes and specific hydroxy and hydroperoxy arachidonic acid derivatives that are potent mediators of neuroinflammation $[35,48]$. There is therefore no doubt that alterations in fatty acid saturation profile may have an impact on neurobiology at multiple levels in addition to regulating susceptibility to peroxidation.

From a clinical perspective, some evidence suggests that diets that are high in n-3 PUFAs may reduce PD risk [49]. Although other studies have found that consumption of n-3 (and n6) PUFAs is not associated with PD risk [50]. Intriguingly, higher consumption of arachidonic acid was found to be correlated with increased PD risk [50]. Although it is possible that diets deficient in certain PUFAs may predispose towards PD, at present there is no clear evidence that n-3 PUFAs are reduced in the PD brain nor that dietary supplementation can be used to treat or prevent this disease. Indeed our data show that the 
relative contribution of PUFAs to the total fatty acid pool is significantly increased in the PD ACC but not altered in the pathologically-spared OCC. Since we have recently documented that neuronal loss does not occur in these early Braak stage cases [51], the alterations in fatty acid composition we have detected are not likely to be related to neuronal loss per se.

A previous study by Julien et al. used a similar approach to us in order to assess global changes in fatty acid profiles in postmortem PD brain tissue [52]. In that study, the temporal cortex was assessed. The temporal cortex is a region that is relatively spared from Lewy body pathology early in PD, being affected only at late stages $[3,6,7,8]$. The overall fatty acid profiles of the temporal cortex [52] were very similar to the profiles we reported herein for both the ACC and OCC. In the study by Julien et al., only one statistically significant change was detected comparing control and PD temporal cortex samples, i.e., they showed decreased levels of C20:4 in the PD patients [52]. Interestingly, we also found decreased levels of C20:4 in the PD ACC, but not the PD OCC, as compared to controls, possibly suggesting this is the earliest fatty acid change observed in PD. In comparing these studies, we conclude that the temporal cortex may have been studied at an early disease stage when the pathological changes have yet to affect the overall fatty acid profile of the PD cases. Another study by Sharon et al. reported increases in 22:4 and 22:6 fatty acids in PD frontal cortex (Brodman area 8) as compared to control frontal cortex [28]. This is in agreement with our data showing increased PUFAs in the PD ACC (and in particular increased 22:6n-3); although frontal neocortex preferantially accumulates $\alpha$-syn only at end-stage PD $[3,6,7,8]$. Based on the available data, we speculate that changes in global fatty acid profiles may be closely linked to PD-associated increases in $\alpha$-syn. 
In addition to the deleterious consequences of the high PI we have detected in the PD ACC, specific fatty acid changes may have other biological consequences. As mentioned above, previous data suggests that increased dietary levels of arachidonic acid (C20:4n-6) may be associated with increased PD risk [50]. Arachidonic acid may be used as a substrate for the formation of proinflammatory eicosanoids that have been shown to play a role in PD neurodegeneration [53]. The increased levels of arachidonic acid we detected in the PD ACC may provide additional substrate for cyclooxygenase-mediated production of such proinflammatory eicosanoids. Also related to arachidonic acid metabolism, we found that levels of the lipid oxidation marker $\mathrm{F}_{2}$-ISPs were increased in the PD ACC. This is not likely to be due to changes in the absolute amounts of arachidonic acid as these were very similar in the PD ACC and Con ACC samples (Table 1). The most plausible explanation is that the PD ACC is under increased oxidative stress as compared to the Con ACC (and both the Con and PD OCC). This would be in agreement with an increased propensity for oxidative damage as predicted by the higher calculated PI values (Fig 1B).

$\mathrm{F}_{2}$-ISPs are a useful marker of lipid oxidative damage in PD [13,54]. For example, the sum of $\mathrm{F}_{2}$ ISPs and structurally related isofurans (also derived from arachidonic acid oxidation) was increased in the PD SN compared to SN from control patients or from patients with neurodegenerative diseases that do not affect the SN [13]. Furthermore, Fessel et al. previously reported that isofurans were a more sensitive lipid peroxidation marker for the PD $\mathrm{SN}$ [55], whereas Pratico et al. showed there was no difference in $\mathrm{F}_{2}$ ISP levels comparing PD and control cerebral neocortex, i.e., a region pathologically spared until very late in PD [56]. We have not measured isofurans in the present work and it is therefore possible that the levels of lipid oxidation may have been underestimated. 
Independent of the impact that altered fatty acid composition has on PD ACC susceptibility to oxidation, it is also important to consider other interactions between $\alpha$-syn and fatty acids. Due to its apolipoprotein-like behaviour, $\alpha$-syn is well known to bind to free fatty acids and phospholipids [57]. In particular, long chain PUFAs have been shown to associate with $\alpha$-syn and promote its oligomerisation [57,58]. In addition, it has also been shown that $\alpha$-syn can directly regulate neuronal PUFA levels. One example of this relationship comes from the dramatic drop in 22:6n-3 levels detected in brains derived from $\alpha$-syn null mice as compared to wild type mice [28]. The data from our current study cannot be used to determine whether the accumulation of $\alpha$-syn in the PD ACC is driven by interactions with specific PUFAs such as 22:6n-3 or whether, conversely, $\alpha$-syn accumulation alters neuronal lipid homeostasis in such a way that levels of 22:6n-3 and other PUFAs are selectively increased. This is an area that appears to warrant further research.

It is also worth considering how changes in brain fatty acid profiles may relate to lipid peroxidation associated with other neurodegenerative diseases. In addition to PD, lipid peroxidation has been reported to be associated with Alzheimer's disease, Huntington's disease, and amyotrophic lateral sclerosis [59,60,61]. Most research in this area has not focused on the fatty acid profiles of susceptible brain regions prior to development of severe pathology. The general thrust of much work in this filed has therefore been more related to factors such as altered energy metabolism and changes in mitochondrial function that may increase free radical stress in the brain (see [62], for a comprehensive review). There are limited data available, for example, indicating that lipid peroxidation in Alzheimer's disease is associated with reduced PUFA levels [63]; however, the analysis of pathologically affected regions raises the possibility that altered fatty acid composition may be associated with the pathology rather than predisposing towards pathology. Other studies have used a lipidomics 
approach to assess fatty acid composition in Alzheimer's disease tissues as well as in subcortical ischemic vascular dementia postmortem tissues [64,65], but no strong consensus has been found that permits a direct comparison with the data we have presented herein. It would be interesting to assess fatty acid profiles in postmortem tissues derived from the earliest stages of these additional neurodegenerative diseases as well as to compare postmortem tissues from PD subjects both with and without dementia to provide further insights into the changes that may increase specific brain regions to lipid oxidative stress.

Like all studies of human postmortem brain tissues, we acknowledge that PMI may contribute to the final lipid profiles we have measured. However, this is unlikely to contribute to the differences we detected as the PMI values were not significantly different between the Con and PD groups.

In conclusion, we provide evidence that the global composition of fatty acids in the PD ACC is altered in a way that increases susceptibility to peroxidation. This has important implications for the eitiology of PD (and possibly other synucleinopathies), particularly as it relates to the PD-oxidative stress hypothesis.

\section{ACKNOWLEDGEMENTS}

Tissues were received from the Sydney Brain Bank at Neuroscience Research Australia and the New South Wales Tissue Resource Centre at the University of Sydney which are supported by the National Health and Medical Research Council of Australia (NHMRC), University of New South Wales, Neuroscience Research Australia, Schizophrenia Research Institute and National Institute of Alcohol Abuse and Alcoholism (NIH (NIAAA) R24AA012725). Professor Paul Else is acknowledged for providing access to gas 
chromatography equipment. This research was supported by the National Health and Medical Research Council of Australia (Project grant \#1008307 to Glenda M. Halliday and Brett Garner, Fellowship \#630434 to Glenda M. Halliday, and Fellowship \#630445 to Brett Garner).

\section{CONFLICT OF INTEREST}

The authors have no conflict of interest to report.

\section{REFERENCES}

[1] Fahn S (2003) Description of Parkinson's disease as a clinical syndrome. Ann N Y Acad Sci 991, 1-14.

[2] Obeso JA, Rodriguez-Oroz MC, Goetz CG, Marin C, Kordower JH, Rodriguez M, Hirsch EC, Farrer M, Schapira AH, Halliday G (2010) Missing pieces in the Parkinson's disease puzzle. Nat Med 16, 653-661.

[3] Dickson DW, Braak H, Duda JE, Duyckaerts C, Gasser T, Halliday GM, Hardy J, Leverenz JB, Del Tredici K, Wszolek ZK, Litvan I (2009) Neuropathological assessment of Parkinson's disease: refining the diagnostic criteria. Lancet Neurol $\mathbf{8}$, $1150-1157$.

[4] Gai WP, Yuan HX, Li XQ, Power JT, Blumbergs PC, Jensen PH (2000) In situ and in vitro study of colocalization and segregation of alpha-synuclein, ubiquitin, and lipids in Lewy bodies. Exp Neurol 166, 324-333.

[5] Halliday GM, Ophof A, Broe M, Jensen PH, Kettle E, Fedorow H, Cartwright MI, Griffiths FM, Shepherd CE, Double KL (2005) Alpha-synuclein redistributes to 
neuromelanin lipid in the substantia nigra early in Parkinson's disease. Brain 128, 2654-2664.

[6] Braak H, Del Tredici K, Rub U, de Vos RA, Jansen Steur EN, Braak E (2003) Staging of brain pathology related to sporadic Parkinson's disease. Neurobiol Aging 24, 197211.

[7] Dickson DW, Fujishiro H, Orr C, DelleDonne A, Josephs KA, Frigerio R, Burnett M, Parisi JE, Klos KJ, Ahlskog JE (2009) Neuropathology of non-motor features of Parkinson disease. Parkinsonism Relat Disord 15 Suppl 3, S1-5.

[8] Halliday GM, McCann H (2010) The progression of pathology in Parkinson's disease. Ann N Y Acad Sci 1184, 188-195.

[9] Fahn S, Cohen G (1992) The oxidant stress hypothesis in Parkinson's disease: evidence supporting it. Ann Neurol 32, 804-812.

[10] Selley ML (1998) (E)-4-hydroxy-2-nonenal may be involved in the pathogenesis of Parkinson's disease. Free Radic Biol Med 25, 169-174.

[11] Jenner P (2003) Oxidative stress in Parkinson's disease. Ann Neurol 53 Suppl 3, S2636; discussion S36-28.

[12] Danielson SR, Andersen JK (2008) Oxidative and nitrative protein modifications in Parkinson's disease. Free Radic Biol Med 44, 1787-1794.

[13] Montine KS, Quinn JF, Zhang J, Fessel JP, Roberts LJ, 2nd, Morrow JD, Montine TJ (2004) Isoprostanes and related products of lipid peroxidation in neurodegenerative diseases. Chem Phys Lipids 128, 117-124.

[14] Ruiperez V, Darios F, Davletov B (2010) Alpha-synuclein, lipids and Parkinson's disease. Prog Lipid Res 49, 420-428.

[15] Farooqui T, Farooqui AA (2011) Lipid-mediated oxidative stress and inflammation in the pathogenesis of Parkinson's disease. Parkinsons Dis 2011, 247467. 
[16] Berg D, Gerlach M, Youdim MB, Double KL, Zecca L, Riederer P, Becker G (2001) Brain iron pathways and their relevance to Parkinson's disease. J Neurochem 79, 225236.

[17] Double KL, Gerlach M, Schunemann V, Trautwein AX, Zecca L, Gallorini M, Youdim MB, Riederer P, Ben-Shachar D (2003) Iron-binding characteristics of neuromelanin of the human substantia nigra. Biochem Pharmacol 66, 489-494.

[18] Double KL, Zecca L, Costi P, Mauer M, Griesinger C, Ito S, Ben-Shachar D, Bringmann G, Fariello RG, Riederer P, Gerlach M (2000) Structural characteristics of human substantia nigra neuromelanin and synthetic dopamine melanins. J Neurochem 75, 2583-2589.

[19] Jenner P (1998) Oxidative mechanisms in nigral cell death in Parkinson's disease. Mov Disord 13 Suppl 1, 24-34.

[20] Garcia-Garcia A, Zavala-Flores L, Rodriguez-Rocha H, Franco R (2012) Thiol-redox signaling, dopaminergic cell death, and Parkinson's disease. Antiox Redox Signal 17, 1764-1784.

[21] Sutachan JJ, Casas Z, Albarracin SL, Stab BR, 2nd, Samudio I, Gonzalez J, Morales L, Barreto GE (2012) Cellular and molecular mechanisms of antioxidants in Parkinson's disease. Nutr Neurosci 15, 120-126.

[22] Dexter DT, Carayon A, Javoy-Agid F, Agid Y, Wells FR, Daniel SE, Lees AJ, Jenner P, Marsden CD (1991) Alterations in the levels of iron, ferritin and other trace metals in Parkinson's disease and other neurodegenerative diseases affecting the basal ganglia. Brain 114, 1953-1975.

[23] Dexter DT, Carter CJ, Wells FR, Javoy-Agid F, Agid Y, Lees A, Jenner P, Marsden CD (1989) Basal lipid peroxidation in substantia nigra is increased in Parkinson's disease. J Neurochem 52, 381-389. 
[24] Venkateshappa C, Harish G, Mythri RB, Mahadevan A, Bharath MM, Shankar SK (2012) Increased oxidative damage and decreased antioxidant function in aging human substantia nigra compared to striatum: implications for Parkinson's disease. Neurochem Res 37, 358-369.

[25] Mythri RB, Venkateshappa C, Harish G, Mahadevan A, Muthane UB, Yasha TC, Srinivas Bharath MM, Shankar SK (2011) Evaluation of markers of oxidative stress, antioxidant function and astrocytic proliferation in the striatum and frontal cortex of Parkinson's disease brains. Neurochem Res 36, 1452-1463.

[26] Turnbull S, Tabner BJ, El-Agnaf OM, Moore S, Davies Y, Allsop D (2001) alphaSynuclein implicated in Parkinson's disease catalyses the formation of hydrogen peroxide in vitro. Free Radic Biol Med 30, 1163-1170.

[27] Giasson BI, Ischiropoulos H, Lee VM, Trojanowski JQ (2002) The relationship between oxidative/nitrative stress and pathological inclusions in Alzheimer's and Parkinson's diseases. Free Radic Biol Med 32, 1264-1275.

[28] Sharon R, Bar-Joseph I, Mirick GE, Serhan CN, Selkoe DJ (2003) Altered fatty acid composition of dopaminergic neurons expressing alpha-synuclein and human brains with alpha-synucleinopathies. J Biol Chem 278, 49874-49881.

[29] Zhu M, Qin ZJ, Hu D, Munishkina LA, Fink AL (2006) Alpha-synuclein can function as an antioxidant preventing oxidation of unsaturated lipid in vesicles. Biochemistry (Mosc) 45, 8135-8142.

[30] Leong SL, Pham CL, Galatis D, Fodero-Tavoletti MT, Perez K, Hill AF, Masters CL, Ali FE, Barnham KJ, Cappai R (2009) Formation of dopamine-mediated alphasynuclein-soluble oligomers requires methionine oxidation. Free Radic Biol Med 46, $1328-1337$. 
[31] Holman RT (1954) Autoxidation of fats and related substances. Prog Chem Fats other Lipids 2, 51-98.

[32] Cosgrove JP, Church DF, Pryor WA (1987) The kinetics of the autoxidation of polyunsaturated fatty acids. Lipids 22, 299-304.

[33] Hulbert AJ (2005) On the importance of fatty acid composition of membranes for aging. J Theor Biol 234, 277-288.

[34] Hulbert AJ, Kelly MA, Abbott SK (2014) Polyunsaturated fats, membrane lipids and animal longevity. J Comp Physiol B 184, 149-166.

[35] Bousquet M, Calon F, Cicchetti F (2011) Impact of omega-3 fatty acids in Parkinson's disease. Ageing Res Rev 10, 453-463.

[36] Abbott SK, Li H, Sanz Muñoz S, Knoch B, Batterham M, Murphy KE, Halliday GM, Garner B (2014) Altered ceramide acyl chain length and ceramide synthase gene expression in Parkinson's disease. Mov Disord 29, 518-526.

[37] Bhatia S, Jenner AM, Li H, Ruberu K, Spiro AS, Shepherd CE, Kril JJ, Kain N, Don A, Garner B (2013) Increased apolipoprotein D dimer formation in Alzheimer's disease hippocampus is associated with lipid conjugated diene levels. $J$ Alzheimers Dis 35, 475-486.

[38] Halliday G, Ng T, Rodriguez M, Harding A, Blumbergs P, Evans W, Fabian V, Fryer J, Gonzales M, Harper C, Kalnins R, Masters CL, McLean C, Milder DG, Pamphlett R, Scott G, Tannenberg A, Kril J (2002) Consensus neuropathological diagnosis of common dementia syndromes: testing and standardising the use of multiple diagnostic criteria. Acta Neuropathol 104, 72-78.

[39] Murphy KE, Cottle L, Gysbers AM, Cooper AA, Halliday GM (2013) ATP13A2 (PARK9) protein levels are reduced in brain tissue of cases with Lewy bodies. Acta Neuropathol Commun 1, 11. 
[40] Abbott SK, Jenner AM, Mitchell TW, Brown SH, Halliday GM, Garner B (2013) An improved high-throughput lipid extraction method for the analysis of human brain lipids. Lipids 48, 307-318.

[41] Abbott SK, Else PL, Atkins TA, Hulbert AJ (2012) Fatty acid composition of membrane bilayers: importance of diet polyunsaturated fat balance. Biochim Biophys Acta 1818, 1309-1317.

[42] Benjamini Y, Hochberg Y (1995) Controlling the false discovery rate: a practical and powerful approach to multiple testing. Journal of the Royal Statistical Society Series $B$ 57, 289-300.

[43] Team RDC (2008) R: A language and environment for statistical computing. $R$ Foundation for Statistical Computing, Vienna, Austria., ISBN 3-900051-900007900050, URL http://www.R-project.org.

[44] Lue LF, Walker DG, Adler CH, Shill H, Tran H, Akiyama H, Sue LI, Caviness J, Sabbagh MN, Beach TG (2012) Biochemical increase in phosphorylated alphasynuclein precedes histopathology of Lewy-type synucleinopathies. Brain Pathol 22, $745-756$.

[45] Zhou J, Broe M, Huang Y, Anderson JP, Gai WP, Milward EA, Porritt M, Howells D, Hughes AJ, Wang X, Halliday GM (2011) Changes in the solubility and phosphorylation of alpha-synuclein over the course of Parkinson's disease. Acta Neuropathol 121, 695-704.

[46] Sayre LM, Smith MA, Perry G (2001) Chemistry and biochemistry of oxidative stress in neurodegenerative disease. Curr Med Chem 8, 721-738.

[47] Huttner WB, Schmidt A (2000) Lipids, lipid modification and lipid-protein interaction in membrane budding and fission--insights from the roles of endophilin A1 and synaptophysin in synaptic vesicle endocytosis. Curr Opin Neurobiol 10, 543-551. 
[48] Sanchez-Mejia RO, Mucke L (2010) Phospholipase A2 and arachidonic acid in Alzheimer's disease. Biochim Biophys Acta 1801, 784-790.

[49] de Lau LM, Bornebroek M, Witteman JC, Hofman A, Koudstaal PJ, Breteler MM (2005) Dietary fatty acids and the risk of Parkinson disease: the Rotterdam study. Neurology 64, 2040-2045.

[50] Miyake Y, Sasaki S, Tanaka K, Fukushima W, Kiyohara C, Tsuboi Y, Yamada T, Oeda T, Miki T, Kawamura N, Sakae N, Fukuyama H, Hirota Y, Nagai M (2010) Dietary fat intake and risk of Parkinson's disease: a case-control study in Japan. $J$ Neurol Sci 288, 117-122.

[51] Murphy KE, Gysbers AM, Abbott SK, Tayebi N, Kim WS, Sidransky E, Cooper A, Garner B, Halliday GM (2014) Reduced glucocerebrosidase is associated with increased alpha-synuclein in sporadic Parkinson's disease. Brain 137, 834-848.

[52] Julien C, Berthiaume L, Hadj-Tahar A, Rajput AH, Bedard PJ, Di Paolo T, Julien P, Calon F (2006) Postmortem brain fatty acid profile of levodopa-treated Parkinson disease patients and parkinsonian monkeys. Neurochem Int 48, 404-414.

[53] Teismann P, Tieu K, Choi DK, Wu DC, Naini A, Hunot S, Vila M, Jackson-Lewis V, Przedborski S (2003) Cyclooxygenase-2 is instrumental in Parkinson's disease neurodegeneration. Proc Natl Acad Sci U S A 100, 5473-5478.

[54] Seet RC, Lee CY, Lim EC, Tan JJ, Quek AM, Chong WL, Looi WF, Huang SH, Wang H, Chan YH, Halliwell B (2010) Oxidative damage in Parkinson disease: Measurement using accurate biomarkers. Free Radic Biol Med 48, 560-566.

[55] Fessel JP, Hulette C, Powell S, Roberts LJ, 2nd, Zhang J (2003) Isofurans, but not F2isoprostanes, are increased in the substantia nigra of patients with Parkinson's disease and with dementia with Lewy body disease. J Neurochem 85, 645-650. 
[56] Pratico D, Lee VM-Y, Trojanowski JQ, Rokach J, Fitzgerald GA (1998) Increased F2-isoprostanes in Alzheimer's disease: evidence for enhanced lipid peroxidation in vivo. FASEB J 12, 1777-1783.

[57] Perrin RJ, Woods WS, Clayton DF, George JM (2001) Exposure to long chain polyunsaturated fatty acids triggers rapid multimerization of synucleins. J Biol Chem 276, 41958-41962.

[58] Sharon R, Bar-Joseph I, Frosch MP, Walsh DM, Hamilton JA, Selkoe DJ (2003) The formation of highly soluble oligomers of alpha-synuclein is regulated by fatty acids and enhanced in Parkinson's disease. Neuron 37, 583-595.

[59] Markesbery WR, Lovell MA (1998) Four-hydroxynonenal, a product of lipid peroxidation, is increased in the brain in Alzheimer's disease. Neurobiol Aging 19, 3336.

[60] Browne SE, Ferrante RJ, Beal MF (1999) Oxidative stress in Huntington's disease. Brain Pathol 9, 147-163.

[61] Pedersen WA, Fu W, Keller JN, Markesbery WR, Appel S, Smith RG, Kasarskis E, Mattson MP (1998) Protein modification by the lipid peroxidation product 4hydroxynonenal in the spinal cords of amyotrophic lateral sclerosis patients. Ann Neurol 44, 819-824.

[62] Reed TT (2011) Lipid peroxidation and neurodegenerative disease. Free Radic Biol Med 51, 1302-1319.

[63] Markesbery WR (1997) Oxidative stress hypothesis in Alzheimer's disease. Free Radic Biol Med 23, 134-147.

[64] Fraser T, Tayler H, Love S (2010) Fatty acid composition of frontal, temporal and parietal neocortex in the normal human brain and in Alzheimer's disease. Neurochem Res 35, 503-513. 
[65] Lam SM, Wang Y, Duan X, Wenk MR, Kalaria RN, Chen CP, Lai MK, Shui G (2014) The brain lipidomes of subcortical ischemic vascular dementia and mixed dementia. Neurobiol Aging 35, 2369-2381. 
Table 1. Anterior cingulate cortex fatty acid composition (nmol / mg tissue)

\begin{tabular}{|c|c|c|c|c|c|c|}
\hline \multirow[b]{2}{*}{ Fatty acid } & \multicolumn{2}{|c|}{$\begin{array}{l}\text { Control } \\
(n=10)\end{array}$} & \multicolumn{2}{|c|}{$\begin{array}{c}\text { PD } \\
(n=9)\end{array}$} & \multirow[t]{2}{*}{$P$-value } & \multirow[t]{2}{*}{$\begin{array}{l}\text { Adjusted } \\
P \text {-value }\end{array}$} \\
\hline & mean & SE & mean & SE & & \\
\hline $16: 0$ & 14.96 & 0.51 & 13.95 & 0.72 & 0.259 & 0.299 \\
\hline $16: 1 \mathrm{n} 7$ & 0.76 & 0.04 & 0.56 & 0.05 & $0.007 \downarrow$ & $0.014 \downarrow$ \\
\hline 18:0 & 22.51 & 1.08 & 17.69 & 1.51 & $0.017 \downarrow$ & $0.024 \downarrow$ \\
\hline $18: 1 \mathrm{n} 9$ & 29.16 & 1.67 & 17.37 & 2.62 & $0.001 \downarrow$ & $0.004 \downarrow$ \\
\hline $18: 1 \mathrm{n} 7$ & 5.37 & 0.27 & 3.80 & 0.41 & $0.005 \downarrow$ & $0.011 \downarrow$ \\
\hline $18: 2 n 6$ & 0.63 & 0.04 & 0.69 & 0.05 & 0.361 & 0.386 \\
\hline $20: 1 n 9$ & 2.86 & 0.28 & 1.25 & 0.28 & $0.001 \downarrow$ & $0.004 \downarrow$ \\
\hline $20: 3 n 6$ & 1.04 & 0.06 & 0.72 & 0.08 & $0.005 \downarrow$ & $0.011 \downarrow$ \\
\hline $20: 4 n 6$ & 6.01 & 0.26 & 6.07 & 0.19 & 0.849 & 0.849 \\
\hline $22: 2 n 6$ & 0.92 & 0.07 & 0.40 & 0.12 & $0.001 \downarrow$ & $0.004 \downarrow$ \\
\hline $22: 4 n 6$ & 6.43 & 0.31 & 4.73 & 0.52 & $0.011 \downarrow$ & $0.018 \downarrow$ \\
\hline $24: 0$ & 2.73 & 0.23 & 1.22 & 0.33 & $0.001 \downarrow$ & $0.004 \downarrow$ \\
\hline $22: 5 n 6$ & 0.58 & 0.06 & 0.86 & 0.09 & $0.018 \uparrow$ & $0.024 \uparrow$ \\
\hline $24: 1 n 9$ & 10.52 & 0.77 & 4.90 & 1.26 & $0.001 \downarrow$ & $0.004 \downarrow$ \\
\hline $22: 6 n 3$ & 6.30 & 0.58 & 7.64 & 0.47 & 0.094 & 0.118 \\
\hline Total & 112.85 & 5.05 & 82.98 & 8.06 & $0.005 \downarrow$ & \\
\hline
\end{tabular}

Values expressed as mean \pm SE. "P-values" are derived from t-test and "Adjusted P-values" are derived from post hoc adjustment for multiple comparisons using the Benjamini and Hochberg approach. The arrows indicate direction of change for statistically significant $(\mathrm{P}<0.05)$ differences. PD, Parkinson's disease. 
Table 2. Occipital cortex fatty acid composition (nmol / mg tissue)

\begin{tabular}{|c|c|c|c|c|c|c|}
\hline \multirow[b]{2}{*}{ Fatty acid } & \multicolumn{2}{|c|}{$\begin{array}{l}\text { Control } \\
(n=10)\end{array}$} & \multicolumn{2}{|c|}{$\begin{array}{c}\text { PD } \\
(n=9)\end{array}$} & \multirow[t]{2}{*}{$P$-value } & \multirow[t]{2}{*}{$\begin{array}{l}\text { Adjusted } \\
P \text {-value }\end{array}$} \\
\hline & mean & SE & mean & SE & & \\
\hline $16: 0$ & 15.22 & 0.56 & 13.49 & 0.67 & 0.065 & 0.231 \\
\hline $16: 1 \mathrm{n} 7$ & 0.62 & 0.03 & 0.48 & 0.03 & $0.003 \downarrow$ & 0.051 \\
\hline $18: 0$ & 20.26 & 1.62 & 16.94 & 1.05 & 0.112 & 0.231 \\
\hline $18: 1 \mathrm{n} 9$ & 22.19 & 3.14 & 15.80 & 1.52 & 0.090 & 0.231 \\
\hline $18: 1 \mathrm{n} 7$ & 4.38 & 0.39 & 3.55 & 0.27 & 0.104 & 0.231 \\
\hline $18: 2 n 6$ & 0.83 & 0.05 & 0.79 & 0.06 & 0.629 & 0.674 \\
\hline $20: 1 n 9$ & 2.29 & 0.46 & 1.31 & 0.22 & 0.078 & 0.231 \\
\hline $20: 3 n 6$ & 0.86 & 0.08 & 0.66 & 0.05 & 0.059 & 0.231 \\
\hline $20: 4 n 6$ & 5.58 & 0.25 & 5.18 & 0.40 & 0.395 & 0.494 \\
\hline $22: 2 n 6$ & 0.52 & 0.15 & 0.30 & 0.07 & 0.215 & 0.309 \\
\hline $22: 4 n 6$ & 4.87 & 0.62 & 3.96 & 0.32 & 0.226 & 0.309 \\
\hline $24: 0$ & 1.74 & 0.52 & 0.87 & 0.16 & 0.139 & 0.231 \\
\hline $22: 5 n 6$ & 0.72 & 0.02 & 0.69 & 0.03 & 0.467 & 0.539 \\
\hline $24: 1 \mathrm{n} 9$ & 7.46 & 1.74 & 4.33 & 0.77 & 0.125 & 0.231 \\
\hline $22: 6 n 3$ & 9.25 & 0.80 & 9.14 & 0.59 & 0.919 & 0.919 \\
\hline Total & 98.37 & 8.85 & 78.63 & 4.61 & 0.073 & \\
\hline
\end{tabular}

Values expressed as mean \pm SE. "P-values" are derived from t-test and "Adjusted P-values" are derived from post hoc adjustment for multiple comparisons using the Benjamini and Hochberg approach. The arrows indicate direction of change for statistically significant $(\mathrm{P}<0.05)$ differences. PD, Parkinson's disease. 


\section{FIGURE LEGENDS}

Figure 1. Comparison of fatty acyl chain structures in control and PD brain samples. Control $(\mathrm{n}=10)$ and PD $(\mathrm{n}=9)$ tissues were collected from the anterior cingulate cortex (ACC) and lipids were extracted, the fatty acid fraction was methylated, and the fatty acid methyl esters were quantified using GC analysis and comparison to internal standards. The data indicates the relative percentage (mol\%) composition that each of the indicated fatty acyl chain structures contributes to the global fatty acid molecular species profile (A). The relative proportion of saturated (SFA), monounsaturated (MUFA) and polyunsaturated (PUFA) fatty acids is also provided along with a further breakdown of the data to reflect changes in n-6 and n-3 PUFA composition (B). The peroxidation index (PI) reflects the theoretical susceptibility of the tissue oxidation (B). Control samples are indicated by the grey bars and PD samples are indicated by the black bars. Values are mean $\pm \mathrm{SE} .{ }^{* *} \mathrm{P}<0.01$ using t-test. Values above bars indicate \% change. All results remained significant when adjusting for multiple comparisons using the Benjamini and Hochberg method.

Figure 2. Comparison of fatty acyl chain structures in control and PD brain samples. Control $(n=10)$ and PD $(n=9)$ tissues were collected from the occipital cortex (OCC) and lipids were extracted, the fatty acid fraction was methylated, and the fatty acid methyl esters were quantified using GC analysis and comparison to internal standards. The data indicates the relative percentage (mol\%) composition that each of the indicated fatty acyl chain structures contributes to the global fatty acid molecular species profile (A). The relative proportion of saturated (SFA), monounsaturated (MUFA) and polyunsaturated (PUFA) fatty acids is also provided along with a further breakdown of the data to reflect changes in n-6 and n-3 PUFA composition (B). The peroxidation index (PI) reflects the theoretical susceptibility 
of the tissue oxidation (B). Control samples are indicated by the grey bars and PD samples are indicated by the black bars. Values are mean $\pm \mathrm{SE}$.

Figure 3. Comparison of $F_{2}$-isoprostane levels in control and PD brain samples. Control $(n=10)$ and PD $(n=9)$ tissues were collected from the anterior cingulate cortex (ACC) and lipids were extracted, the $\mathrm{F}_{2}$-isoprostane fraction was purified by solid phase extraction. $\mathrm{F}_{2}$ isoprostane PFBenzyl esters were prepared and derivatised with BSTFA and TMCS for analysis using GC-MS. The $\mathrm{F}_{2}$-isoprostane quantified by comparison to internal standards. Data are expressed as ng $\mathrm{F}_{2}$-isoprostane per $\mathrm{g}$ of tissue. Control samples are indicated by the grey bars and PD samples are indicated by the black bars. Values are mean \pm SE. Values above bars indicate $\%$ change. $* \mathrm{P}<0.05$ using one-way ANOVA with post-hoc testing by Fisher's LSD. 


\section{Abbott et al. Fig 1}

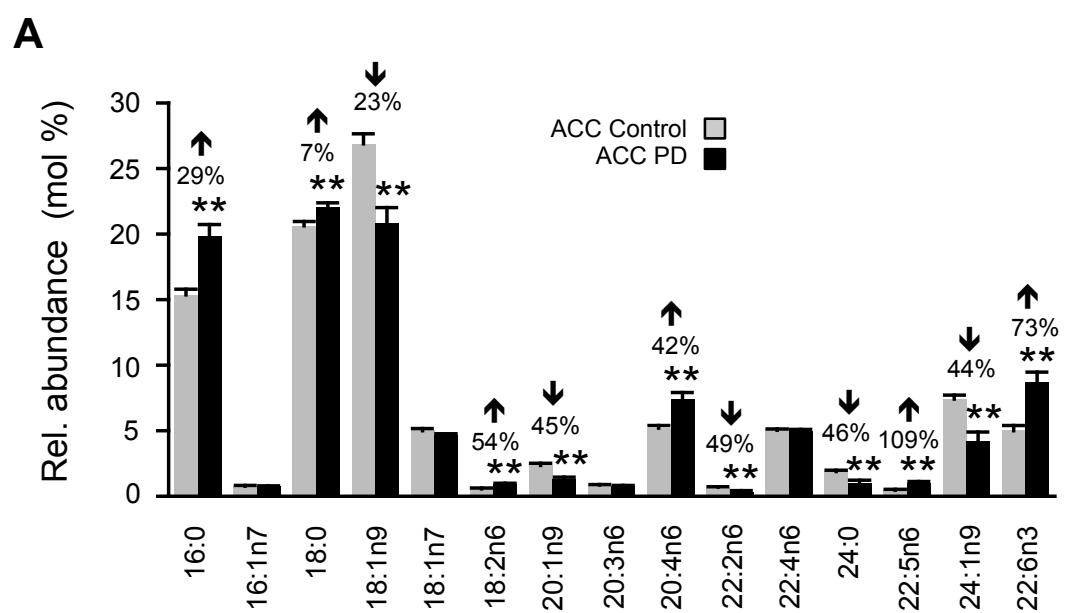

B

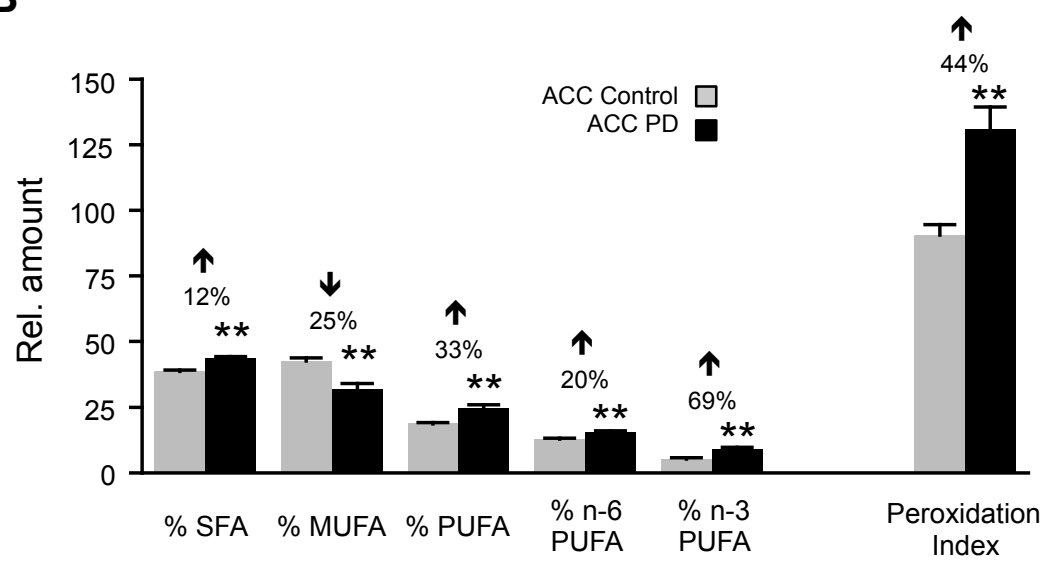




\section{Abbott et al. Fig 2}

A

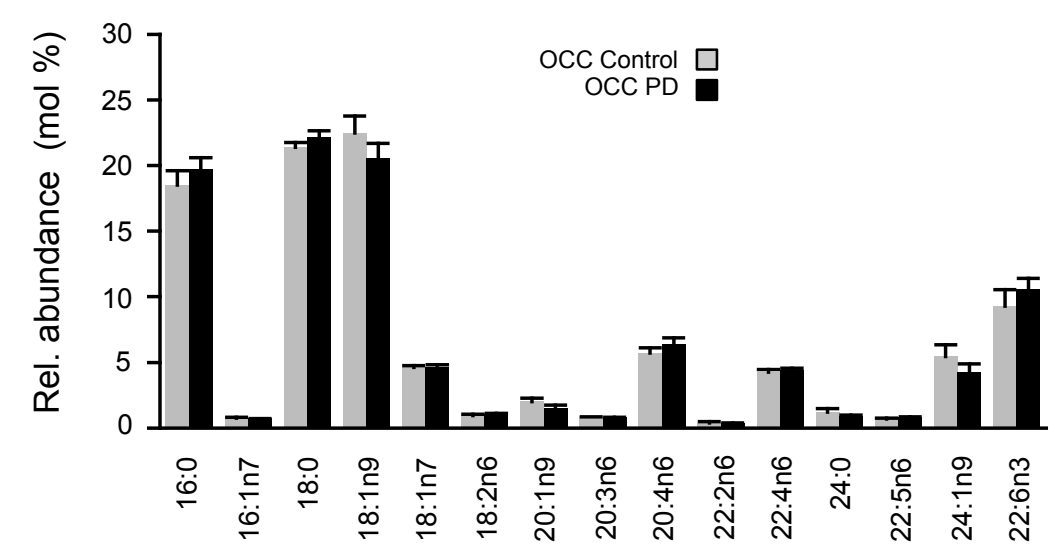

B

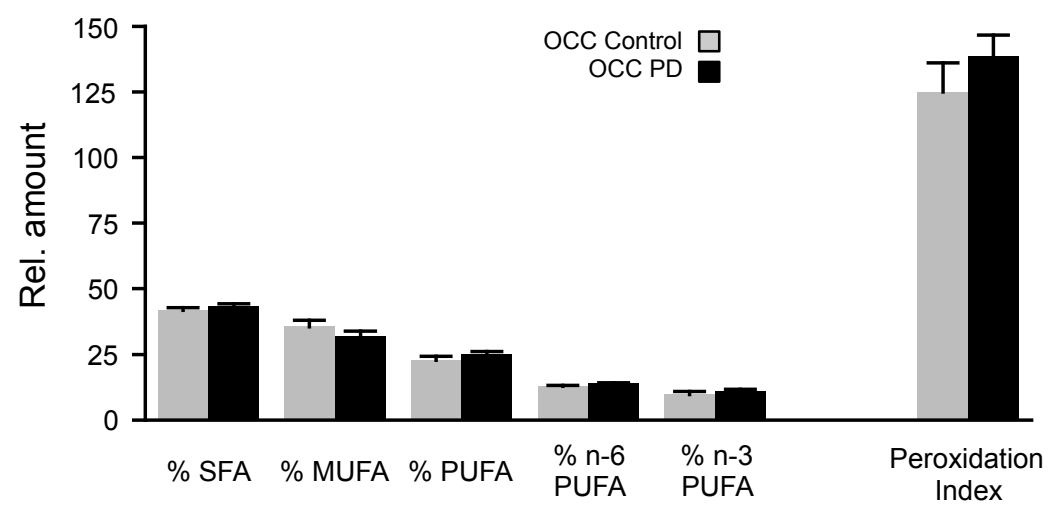


Abbott et al. Fig 3

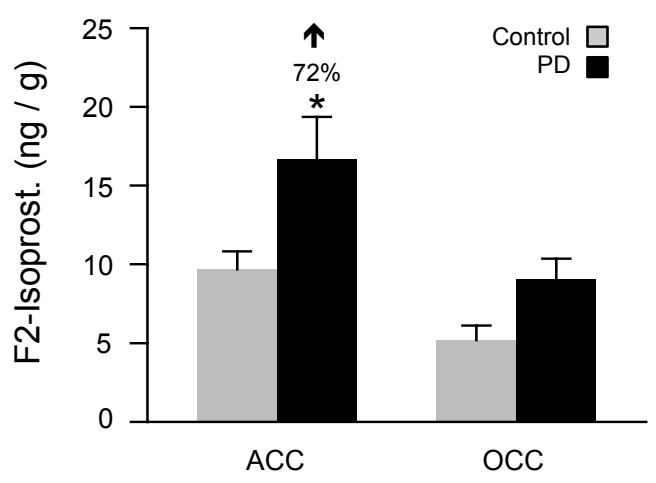

